\title{
EFEITOS DE EXERCÍCIOS PARA ASSOALHO PÉLVICO EM MULHERES IDOSAS DE DIFERENTES NÍVEIS SOCIOECONÔMICOS
}

\section{Juliana Schulze Burti}

Fisioterapeuta e docente de Educação Física, Doutora em Psicologia Social (PUC-SP) Mestre em Ciências da Saúde (Unifesp), Docente e coordenadora de estágios do curso de Fisioterapia da PUC-SP, Brasil.

\section{Sueli Marino}

Psicóloga. Doutoranda pelo Departamento de Psicologia Social da Pontifícia Universidade Católica de São Paulo (PUC-SP), Brasil.

Juliana de Paula da Silva Cruz

Fisioterapeuta, Brasil.

Mary Jane Paris Spink

Docente Titular do Programa de Pós-Graduação em Psicologia Social da Faculdade de Ciências Humanas e Saúde (PUC-SP), Brasil.
RESUMO: Este estudo visa avaliar os efeitos de um programa de exercícios para o assoalho pélvico (AP) em mulheres idosas de diferentes níveis socioeconômicos. Trata-se de estudo experimental longitudinal não controlado. Foram avaliadas 57 mulheres divididas em dois grupos, baixo (B) e alto (A) nível socioeconômico, que participaram de cinco encontros semanais de exercícios em grupo e responderam a questionários estruturados antes e após os encontros. O grupo B tinha maior porcentagem de mulheres não-brancas $(30,4 \%$ versus $5,9 \%$; $\mathrm{p}=$ $0,023)$, com mais dor $(52,9 \%$ versus $82,6 \%$; $p=0,021)$, incontinência urinária $(2,9 \%$ versus $30,4 \% ; \mathrm{p}=0,005)$, e falta de libido que as do grupo A ( $46,9 \%$ versus $75 \%$; $p=0,046)$. Ambos os grupos referiram melhora dos sintomas urinários, consciência corporal e do assoalho pélvico após os exercícios em grupo, sugerindo que essa possa ser uma boa estratégia de abordagem de promoção de saúde em idosas de diferentes níveis socioeconômicos.

PALAVRAS-CHAVE: Assoalho pélvico; Conscientização; Incontinência Urinária; Técnicas de Exercício e de Movimento.

\section{EFFECTS OF PELVIC FLOOR MUSCLES EXERCISES IN ELDERLY FEMALES FROM DIFFERENT SOCIO-ECO- NOMIC BACKGROUNDS}

\begin{abstract}
Effects on the pelvic floor muscles in elderly females from different socio-economic backgrounds are assessed by a non-controlled longitudinal experimental study. Fifty-seven females were evaluated and divided into two groups: low (B) and high (A) socio-economic background, who participated in five weekly meetings involving group exercises and answered structured questionnaires prior to and posterior to meetings. Group B had the highest percentage of non-white females $(30.4 \%$ versus $5.9 \% ; \mathrm{p}=0.023)$, with more pain $(52.9 \%$ versus $82.6 \% ; \mathrm{p}=0.021)$, urinary incontinence $(2.9 \%$ versus $30.4 \% ; \mathrm{p}=$ 0.005 ), and lack of libido, than those of Group A ( $46.9 \%$ versus $75 \%$; $p$ $=0.046)$. The two groups improved urinary symptoms, body and pelvic floor muscles awareness after group exercises. The above suggests that it may be a good strategy in health promotion in the elderly from different socio-economic backgrounds.
\end{abstract}

KEY WORDS: Pelvic floor muscles; Awareness; Urine incontinence; Techniques for exercises and movements. 


\section{INTRODUÇÃO}

Incontinência urinária (IU) é definida como qualquer perda involuntária de urina, que ocorre associada ou não a esforços ${ }^{1}$. Pode ser causada por danos no controle neural da bexiga e/ou nos músculos do assoalho pélvico, ou por traumas mecânicos no assoalho pélvico, como nos casos de partos complicados e cirurgias pélvicas. O risco é acrescido pelo parto vaginal, aumento da idade, paridade, obesidade e menopausa ${ }^{2}$. Incontinência urinária de esforço (IUE) é a perda involuntária de urina que ocorre durante o esforço, como espirro ou tosse; incontinência urinária de urgência (IUU) é a perda involuntária de urina precedida ou associada à urgência miccional e incontinência urinária mista (IUM) é a perda involuntária de urina associada com urgência e esforço ${ }^{2}$.

A IU é considerada uma das mais importantes síndromes geriátricas, apresentando-se como uma condição multifatorial ${ }^{3}$. A queixa de IU em idosas no Brasil é alta de acordo com estudos recentes, que apontam prevalências de $42,4 \%{ }^{4}, 50 \% 5$ e até $57,4 \%{ }^{6}$ gerando problemas sociais e de higiene, que refletem na piora da qualidade de vida dessas mulheres. A condição ainda é negligenciada por profissionais de saúde e há na sociedade uma falsa ideia de que seja um processo natural do envelhecimento ${ }^{7}$. Uma abordagem multiprofissional, que englobe a prevenção, a avaliação e o tratamento da IU é fundamental para um enfrentamento do problema.

Estudos mostram que o desconhecimento sobre tratamentos é frequente na população de mulheres que apresentam incontinência, e que muitas não recebem orientação médica e acham normal perder urina ${ }^{8,9,10}$. Outro aspecto importante é o custo que o problema gera para essas pessoas; estudos americanos sobre os gastos anuais diretos com a IU em todas as idades estimam um valor em torno de 16 bilhões de dólares, ultrapassando custos com câncer de mama, ovário, útero e colo de útero, sendo que as mulheres americanas com IU grave pagam US $\$ 900,00$ por ano para cuidados de rotina, com decréscimo significativo na qualidade de vida relacionada à saúde ${ }^{11,12}$. O tratamento efetivo da IU pode diminuir os gastos e melhorar a qualidade de vida de seus portadores.

Há várias formas para tratar a IU em mulheres, incluindo o tratamento conservador, medicamentos e cirurgias. As intervenções conservadoras geralmente são recomendadas como a primeira forma de tratamento e incluem meios físicos, comportamentais e psicológicos, muitas vezes feitas de forma combinada ${ }^{13}$. A vantagem dessa abordagem é a ausência de efeitos colaterais, o baixo custo e a baixa complexidade.

As terapias físicas são orientadas por profissionais de reabilitação, usando exercícios específicos para recuperar ou melhorar o controle e função dos músculos do assoalho pélvico (MAP). O treinamento muscular do assoalho pélvico envolve a contração e relaxamento voluntário repetitivo e seletivo dos MAP e pode ser ensinado por profissionais de reabilitação, mas também pode ser feito de forma independente e regular por pacientes, melhorando a força, a resistência e a coordenação desses músculos $^{14,15}$.

Sabendo-se que há desconhecimento generalizado sobre a IU e seu tratamento na população geral, e a prevalência deste problema é alta, principalmente em mulheres idosas, e que o tratamento conservador utilizando exercícios específicos é indicado como primeira linha de tratamento, este estudo objetiva verificar se é possível melhorar o conhecimento do assoalho pélvico através de um método de exercícios em grupos de mulheres idosas de diferentes níveis socioeconômicos.

\section{METODOLOGIA}

Estudo experimental, longitudinal, não-controlado, aprovado pelo comitê de ética da Pontifícia Universidade Católica de São Paulo (PUC-SP), número CAAE: 58208116.9.0000.5482, realizado em dois grupos: alto nível socioeconômico (A), pertencente à Universidade Aberta à Maturidade da PUC-SP, e grupo baixo nível socioeconômico (B), pertencente à Paróquia Maria Mãe da Igreja, situada na periferia da zona Sul da cidade de São Paulo. As mulheres de ambos os grupos foram convidadas a participar voluntariamente e assinaram termo de consentimento livre esclarecido. Foram incluídas mulheres acima de 50 anos, que não apresentassem qualquer problema clínico que as impedisse de realizar ou compreender os exercícios.

A definição dos níveis socioeconômicos foi ba- 
seada no Índice de Desenvolvimento Humano (IDH) dos locais onde as mulheres participantes residiam. O IDH foi criado com o objetivo de identificar, de maneira sintética, o nível de desenvolvimento econômico e social alcançado por diferentes países do mundo, mas pode ser falho ao não constatar diferenças importantes, como distribuição de renda, desemprego e violência. Para suprimir tais falhas utilizou-se estudo da Secretaria Municipal do Desenvolvimento, Trabalho e Solidariedade da Prefeitura de São Paulo, que fez levantamento no ano de 2002 obedecendo as informações disponibilizadas pelo IBGE no Censo 2000, pela Fundação SEADE, e pelas Secretarias Municipais de Finanças e de Saúde, que adequou a metodologia de análise do IDH para a realidade paulistana ${ }^{16}$. O estudo mostrou que a cidade sofre profundamente com a desigualdade: apenas seis distritos têm o IDH elevado (acima de 0,8 ) enquanto 38 possuem valores baixos de desenvolvimento humano (abaixo de 0,5$)^{17}$. Para avaliar os grupos de mulheres que participaram do presente estudo foram utilizados os dados desse levantamento, que reflete de maneira mais real a cidade de São Paulo. As mulheres do grupo A residiam em Pinheiros, Alto de Pinheiros e Perdizes (IDH 0,833, 0,801 e 0,762), enquanto as do B residiam na região do Jardim São Luís e Jardim Ângela (IDH 0,441 e 0,402), mostrando de forma clara a diferença de nível socioeconômico (Tabela 1).
Foram feitas cinco sessões de exercícios em cada um dos grupos, com duração de 2 horas, ministradas por fisioterapeuta especialista. As sessões consistiam de exercícios para a musculatura do assoalho pélvico de forma isolada e também associados a exercícios respiratórios, de equilíbrio, resistência, coordenação e controle postural. Os exercícios tinham sua dificuldade aumentada ao longo das semanas, conforme as mulheres se adaptavam à execução correta (Quadro 1). Antes da primeira sessão foi aplicado um questionário com perguntas incluindo informações pessoais, dados clínicos e avaliação sobre a percepção corporal e conhecimento do assoalho pélvico. Ao final das cinco sessões as mulheres respondiam novamente algumas questões para análise comparativa. Os questionários eram auto administrados, com exceção das mulheres analfabetas que não conseguiam responder $\mathrm{e}$ recebiam auxílio da pesquisadora, sem interferência nas respostas.

Tabela 1. Resultado calculado do IDH para os bairros estudados da cidade de São Paulo onde residiam as mulheres dos grupos A e B

\begin{tabular}{|c|c|c|c|c|c|c|c|}
\hline Distritos & Posição & IDH & População & Distritos & Posição & IDH & População \\
\hline Grupo A & & & & Grupo B & & & \\
\hline Pinheiros & 4 & 0,833 & 62.997 & & & & \\
\hline Alto de Pinheiros & 6 & 0,801 & 44.454 & & & & \\
\hline \multirow[t]{3}{*}{ Perdizes } & 9 & 0,762 & 102.445 & & & & \\
\hline & & & & Jardim Ângela & 91 & 0,402 & 245.805 \\
\hline & & & & Jardim São Luís & 82 & 0,441 & 239.161 \\
\hline
\end{tabular}

Fonte: IBGE/Censo 2000 e Fundação SEADE. Elaboração SDTS/PMSP ${ }^{17}$. 
Quadro 1. Cronograma das cinco sessões de exercícios para percepção e treinamento do assoalho pélvico

\begin{tabular}{|c|c|}
\hline Semana & Conteúdo \\
\hline 1 & $\begin{array}{l}\text { - } \\
\text { - } \quad \text { Roda de conversa introdutória e resposta aos } \\
\text { questionários } \\
\text { - Introdução ao método com explicações teóricas } \\
\text { sobre anatomia e fisiopatologia das principais } \\
\text { disfunções corporais no envelhecimento } \\
\text { - Exercícios básicos de postura, respiração e assoa- } \\
\text { lho pélvico }\end{array}$ \\
\hline 2 & $\begin{array}{ll}\text { - } & \text { Exercícios de conscientização corporal/postural } \\
\text { - } & \text { Exercícios de respiração e mobilização corporal } \\
\text { global } \\
\text { - } \\
\text { - } \\
\text { - } \\
\end{array}$ \\
\hline 3 & $\begin{array}{l}\text { - Consciência corporal/postura, respiração e mobi- } \\
\text { lização (iguais à semana 2) } \\
\text { - } \quad \text { Exercícios para assoalho pélvico com progressão } \\
\text { de dificuldade (aumento do número de séries e } \\
\text { tempo de contração) } \\
\text { - Exercícios de estabilização de tronco, resistência } \\
\text { e força (ponte) } \\
\text { - } \quad \text { Relaxamento }\end{array}$ \\
\hline 4 & $\begin{array}{l}\text { - } \quad \text { Todos os exercícios da semana } 3 \\
\text { - Exercícios de mobilidade pélvica e de coluna } \\
\text { associados aos exercícios de assoalho pélvico } \\
\text { (coordenação) } \\
\text { - } \quad \text { Relaxamento } \\
\end{array}$ \\
\hline 5 & $\begin{array}{l}\text { - Todos os exercícios da semana } 4 \\
\text { - Treino funcional (aprendizado de como aplicar } \\
\text { os exercícios do método nas atividades de vida } \\
\text { diária) } \\
\text { - } \quad \text { Relaxamento } \\
\text { - } \\
\text { Roda de conversa final e resposta aos questioná- } \\
\text { rios finais }\end{array}$ \\
\hline
\end{tabular}

Para avaliação estatística foi realizada a análise descritiva dos dados com nível de significância de 5\% (p $<0,05$ ). Foi feita comparação das variáveis estudadas entre os dois grupos (A e B). Para as variáveis qualitativas foram utilizados os testes Qui-quadrado e teste Exato de Fisher. As variáveis quantitativas foram submetidas à verificação da normalidade pelo teste de Komolgorov-Smirnov e como não houve distribuição normal foi aplicado o teste não paramétrico de Mann-Whitney. O teste paramétrico t-Student foi utilizado para comparar os grupos com e sem incontinência urinária. Os dados foram digitados em planilhas do Microsoft Excel e analisados pelo programa SPSS versão 22.0 para Windows.

\section{RESULTADOS}

Participaram do estudo 57 mulheres, sendo 34 no grupo A e 23 no grupo B. A idade média das mulheres do grupo A foi de 67,5 anos, e nas mulheres do B de 63 anos.

As sessões de exercícios foram realizadas entre maio e novembro de 2016, iniciadas pelo grupo A, depois o B.

As variáveis cor de pele, presença de dor e cirurgias apresentaram associação estatisticamente significativa entre os grupos, com uma proporção maior de mulheres não brancas no grupo B (30,4\% versus 5,9\%; $\mathrm{p}=0,023)$, proporção superior para a variável presença de dor no grupo B (52,9\% versus $82,6 \%$; $\mathrm{p}=0,021)$, e proporção superior de mulheres que realizaram cirurgia no grupo A (91,2\% versus $60,9 \%$; $=0,009)$ (Tabela 2 ).

Tabela 2. Número e percentual de sujeitos, segundo características demográficas e clínicas

\begin{tabular}{|c|c|c|c|c|c|}
\hline \multirow[b]{2}{*}{ Variáveis } & \multirow[b]{2}{*}{ Categorias } & \multicolumn{2}{|c|}{ Grupos } & \multirow[b]{2}{*}{$\mathrm{p}^{*}$} & \multirow[t]{2}{*}{ Total } \\
\hline & & A & B & & \\
\hline & & n (\%) & n (\%) & & n (\%) \\
\hline \multirow{2}{*}{$\begin{array}{l}\text { Cor da } \\
\text { pele } ¥\end{array}$} & Branca & $32(94,1)$ & $16(69,6)$ & 0,023 & $48(84,2)$ \\
\hline & Não branca & $2(5,9)$ & $7(30,4)$ & & $9(15,8)$ \\
\hline \multirow{3}{*}{ IMC $(\mathrm{kg} / \mathrm{m} 2)$} & Eutrófico & $13(39,4)$ & $10(45,5)$ & 0,564 & $23(41,8)$ \\
\hline & Sobrepeso & $15(45,5)$ & $7(31,8)$ & & $22(40,0)$ \\
\hline & Obeso & $5(15,1)$ & $5(22,7)$ & & $10(18,2)$ \\
\hline \multirow{2}{*}{$\begin{array}{l}\text { Presença de } \\
\text { doenças } ¥\end{array}$} & Não & $6(17,6)$ & $2(8,7)$ & 0,453 & $8(14,0)$ \\
\hline & Sim & $28(82,4)$ & $21(91,3)$ & & $49(86,0)$ \\
\hline \multirow{2}{*}{$\begin{array}{l}\text { Presença de } \\
\text { dor }\end{array}$} & Não & $16(47,1)$ & $4(17,4)$ & 0,021 & $20(35,1)$ \\
\hline & Sim & $18(52,9)$ & $19(82,6)$ & & $37(64,9)$ \\
\hline \multirow{2}{*}{$\begin{array}{l}\text { Acidentes ou } \\
\text { traumas } ¥\end{array}$} & Não & $29(85,3)$ & $20(87,0)$ & 1,000 & $49(86,0)$ \\
\hline & Sim & $5(14,7)$ & $3(13,0)$ & & $8(14,0)$ \\
\hline \multirow{2}{*}{ Cirurgia $¥$} & Não & $3(8,8)$ & $9(39,1)$ & 0,009 & $12(21,1)$ \\
\hline & Sim & $31(91,2)$ & $14(60,9)$ & & $45(78,9)$ \\
\hline \multirow{3}{*}{$\begin{array}{l}\text { Medicamen- } \\
\text { tos } ¥\end{array}$} & Não & $7(20,6)$ & $5(21,7)$ & 1,000 & $12(21,1)$ \\
\hline & Sim & $27(79,4)$ & $18(78,3)$ & & $45(78,9)$ \\
\hline & Total & $\begin{array}{c}34 \\
(100,0)\end{array}$ & $\begin{array}{c}23 \\
(100,0)\end{array}$ & & $57(100,0)$ \\
\hline
\end{tabular}

*Qui-quadrado; $¥$ teste Exato de Fisher. 
Metade das mulheres $(50,87 \%)$ apresentava incontinência urinária (IU) e 33,33\% apresentavam constipação intestinal. Houve associação estatisticamente significativa entre as variáveis incontinência urinária de esforço (20,6\% versus 52,2\%; $\mathrm{p}=0,013)$, incontinência urinária mista $(2,9 \%$ versus $30,4 \% ; \mathrm{p}=0,005)$ e constipação intestinal $(14,7 \%$ versus $52,2 \% ; p=0,002)$ nos grupos: mulheres do grupo B apresentaram percentual superior para todos estes eventos quando comparadas ao grupo A. A quantidade de urina perdida, uso de absorventes, presença de incontinência fecal, incontinência urinária de urgência e idas frequentes ao banheiro (polaciúria) são sintomas que não apresentaram diferença entre os grupos.

Em relação à variável IMC, as mulheres obesas têm probabilidade superior de apresentarem IU quando comparadas às mulheres eutróficas, respectivamente $\mathrm{p}=$ 0,045 e $\mathrm{p}=0,041$.

Em relação à história obstétrica, houve maior proporção de partos do tipo fórceps e abortos entre as mulheres do grupo B, respectivamente, $\mathrm{p}=0,016 \mathrm{e} \mathrm{p}=$ 0,019. Quanto à questão da falta de libido, as mulheres do grupo B também apresentaram proporção superior em relação ao grupo A $(p=0,046)$ (Tabela 3).

Tabela 3. Características obstétricas e sexualidade

\begin{tabular}{|c|c|c|c|c|c|}
\hline \multirow{3}{*}{ Variáveis } & \multirow{3}{*}{ Categorias } & \multicolumn{4}{|c|}{ (Continua) } \\
\hline & & \multicolumn{2}{|c|}{ Grupos } & \multicolumn{2}{|r|}{ Total } \\
\hline & & A & B & $\mathrm{p}^{*}$ & \\
\hline & & $\mathrm{n}(\%)$ & $\mathrm{n}(\%)$ & & $\mathrm{n}(\%)$ \\
\hline \multirow{3}{*}{$\begin{array}{l}\text { Parto } \\
\text { normal }\end{array}$} & Nenhum & $18(56,2)$ & $9(39,1)$ & 0,338 & $27(49,1)$ \\
\hline & 1 a 2 & $7(21,9)$ & $9(39,1)$ & & $16(29,1)$ \\
\hline & $\geq 3$ & $7(21,9)$ & $5(21,8)$ & & $12(21,8)$ \\
\hline \multirow{3}{*}{ Fórceps } & Nenhum & $28(87,5)$ & $15(65,2)$ & 0,016 & $43(78,2)$ \\
\hline & 1 & $2(6,3)$ & $8(34,8)$ & & $10(18,2)$ \\
\hline & $\geq 2$ & $2(6,3)$ & $0(0,0)$ & & $2(3,6)$ \\
\hline \multirow{3}{*}{$\begin{array}{l}\text { Parto } \\
\text { cesárea }\end{array}$} & Nenhum & $11(34,4)$ & $10(43,5)$ & 0,649 & $21(38,2)$ \\
\hline & 1 a 2 & $14(43,8)$ & $10(43,5)$ & & $24(46,6)$ \\
\hline & $\geq 3$ & $7(21,8)$ & $2(13,0)$ & & $10(18,2)$ \\
\hline \multirow{3}{*}{ Aborto } & Nenhum & $28(87,5)$ & $13(56,5)$ & 0,019 & $41(74,6)$ \\
\hline & 1 & $1(3,1)$ & $6(26,1)$ & & $7(12,7)$ \\
\hline & $\geq 2$ & $3(9,4)$ & $4(17,4)$ & & $7(12,7)$ \\
\hline
\end{tabular}

.

\begin{tabular}{|c|c|c|c|c|c|}
\hline \multirow[b]{2}{*}{ Variáveis } & \multirow[b]{2}{*}{ Categorias } & \multicolumn{2}{|c|}{ Grupos } & \multirow[b]{2}{*}{$\mathrm{p}^{*}$} & \multirow[t]{2}{*}{ Total } \\
\hline & & A & B & & \\
\hline & & $\mathrm{n}(\%)$ & $\mathrm{n}(\%)$ & & n (\%) \\
\hline \multirow{2}{*}{$\begin{array}{l}\text { Vida } \\
\text { sexual } \\
\text { ativa }\end{array}$} & Não & $21(61,8)$ & $10(43,5)$ & \multirow[t]{2}{*}{0,174} & $31(54,4)$ \\
\hline & Sim & $13(38,2)$ & $13(56,5)$ & & $26(45,6)$ \\
\hline \multirow{2}{*}{ Orgasmo } & Não & $19(61,3)$ & $7(38,9)$ & \multirow[t]{2}{*}{0,130} & $26(53,1)$ \\
\hline & Sim & $12(38,7)$ & $11(61,1)$ & & $23(46,9)$ \\
\hline \multirow{2}{*}{$\begin{array}{l}\text { Dispareu- } \\
\text { nia } ¥\end{array}$} & Não & $26(89,7)$ & $13(76,5)$ & \multirow[t]{2}{*}{0,397} & $39(84,8)$ \\
\hline & Sim & $3(10,3)$ & $4(23,5)$ & & $7(15,2)$ \\
\hline \multirow{2}{*}{$\begin{array}{l}\text { Falta de } \\
\text { Libido }\end{array}$} & Não & $17(53,1)$ & $5(25,0)$ & \multirow[t]{2}{*}{0,046} & $22(42,3)$ \\
\hline & Sim & $15(46,9)$ & $15(75,0)$ & & $30(57,7)$ \\
\hline \multirow{3}{*}{$\begin{array}{l}\text { Pouca } \\
\text { Lubrifica- } \\
\text { ção }\end{array}$} & Não & $22(71,0)$ & $12(60,0)$ & \multirow[t]{2}{*}{0,417} & $34(66,7)$ \\
\hline & Sim & $9(29,0)$ & $8(40,0)$ & & $17(33,3)$ \\
\hline & Total & $34(100,0)$ & $23(100,0)$ & & $\begin{array}{c}57 \\
(100,0)\end{array}$ \\
\hline
\end{tabular}

*Qui-quadrado; $¥$ teste Exato de Fisher.

Idade, número de gestações, frequência urinária noturna e frequência de relações sexuais mensais não apresentaram diferença entre os grupos. Somente a variável Escala Visual Analógica (EVA) de dor apresentou diferença estatisticamente significativa entre os grupos. Mulheres do grupo B apresentam mediana de 5,0 para dor enquanto que, no grupo $\mathrm{A}$, este valor foi de 1,5 (p $=0,004)$.

$\mathrm{Na}$ comparação entre os questionários inicial e final, a variável sobre conhecimento do assoalho pélvico (AP) apresentou associação estatisticamente significativa. Das mulheres do grupo B, 70,6\% relataram que não sabiam o que era o AP e agora sabem versus 32,4\% do grupo A ( $\mathrm{p}=0,012)$, mostrando que o grupo de baixo nível socioeconômico tinha menor conhecimento a respeito do assunto.

Algumas variáveis avaliadas mostraram resultados positivos para ambos os grupos: das mulheres que apresentavam perdas urinárias, 32,4\% do grupo A e 64,7\% do grupo B acreditam que melhoraram os sintomas; $61,8 \%$ do grupo A e $81,5 \%$ do grupo B conseguiram perceber e controlar melhor a respiração; 94,1\% das mulheres do grupo A e 100\% do B referiram melhora na postura. 
Em relação ao método de exercícios em grupo, $71,6 \%$ das mulheres sugeriram que as aulas tivessem continuidade e 34,2\% consideraram a metodologia ótima ou excelente (Tabela 4).

Tabela 4. Conhecimento corporal após as sessões de ginástica feminina entre os grupos

\begin{tabular}{|c|c|c|c|c|c|}
\hline \multirow[b]{2}{*}{ Variáveis } & \multirow[b]{2}{*}{ Categorias } & \multicolumn{2}{|c|}{ Grupos } & \multirow[b]{2}{*}{$\mathrm{p}^{*}$} & \multirow[t]{2}{*}{ Total } \\
\hline & & A & B & & \\
\hline & & n (\%) & n (\%) & & n (\%) \\
\hline \multirow{3}{*}{$\begin{array}{l}\text { Depois das aulas você } \\
\text { percebeu que: }\end{array}$} & Não sabia o que era AP, agora sei & $11(32,4)$ & $12(70,6)$ & 0,010 & $23(45,1)$ \\
\hline & $\begin{array}{l}\text { Eu sabia o que era o AP, mas agora conhe- } \\
\text { ço mais }\end{array}$ & $23(67,6)$ & $5(29,4)$ & & $28(54,9)$ \\
\hline & $\begin{array}{l}\text { Nada mudou em relação ao meu conhe- } \\
\text { cimento }\end{array}$ & -- & -- & -- & -- \\
\hline \multirow{3}{*}{ Em relação à respiração } & $\begin{array}{l}\text { Agora consigo perceber melhor como } \\
\text { respiro e controlar a respiração }\end{array}$ & $21(61,8)$ & $14(82,4)$ & 0,301 & $35(68,6)$ \\
\hline & $\begin{array}{l}\text { Eu já sabia respirar corretamente, mas } \\
\text { agora consigo controlar melhor ainda }\end{array}$ & $12(35,3)$ & $3(17,6)$ & & $15(29,4)$ \\
\hline & $\begin{array}{l}\text { Nada mudou em relação ao meu conhe- } \\
\text { cimento }\end{array}$ & $1(2,9)$ & $0(0,0)$ & & $1(2,0)$ \\
\hline \multirow{2}{*}{$\begin{array}{l}\text { Em relação à consciência } \\
\text { corporal }\end{array}$} & Melhorei minha consciência corporal & $32(94,1)$ & $17(100,0)$ & 0,547 & $49(96,1)$ \\
\hline & Nada mudou & $2(5,9)$ & $0(0,0)$ & & $2(3,9)$ \\
\hline \multirow[t]{2}{*}{ Em relação à postura } & Melhorei minha postura & $32(94,1)$ & $17(100,0)$ & 0,547 & $49(96,1)$ \\
\hline & Nada mudou & $2(5,9)$ & $0(0,0)$ & & $2(3,9)$ \\
\hline \multirow{3}{*}{$\begin{array}{l}\text { Em relação às perdas } \\
\text { urinárias e ou fecais }\end{array}$} & Eu não tinha perdas & $19(55,9)$ & $5(29,4)$ & 0,089 & $24(47,1)$ \\
\hline & Eu perdia e acho que melhorei & $11(32,4)$ & $11(64,7)$ & & $22(43,1)$ \\
\hline & Eu perdia e continuo na mesma & $4(11,8)$ & $1(5,9)$ & & $5(9,8)$ \\
\hline Total & & $34(100,0)$ & $23(100,0)$ & & $57(100,0)$ \\
\hline
\end{tabular}

*Qui-quadrado; ¥ teste Exato de Fisher; -- categoria não mencionada.

\section{DISCUSSÃO}

A alta prevalência de incontinência urinária na população idosa feminina justifica a busca por estratégias eficazes de promoção de saúde. A falta de conhecimento e os altos custos de tratamentos médicos especializados dificultam o acesso de grande parte da população a esses serviços, principalmente aqueles com piores condições socioeconômicas, o que justifica a busca por estratégias eficazes e viáveis, como abordagem em grupo, por exemplo.

A seguir serão discutidos os principais achados desta pesquisa, realizada em dois grupos de diferentes níveis socioeconômicos, como presença de dor, incontinência urinária, obesidade, falta de libido e repercussão do programa de exercícios em grupo para assoalho pélvico, postura, respiração e consciência corporal.

DOR

A presença de dor foi um dado marcante nos grupos: $52,9 \%$ das mulheres do grupo A (alto nível) e $82,6 \%$ do grupo B (baixo nível) relataram sentir algum tipo de dor, sendo que esta foi significativamente mais intensa no grupo B. Esse é um dado comum em estudos nacionais e internacionais. Pesquisa feita na cidade de Salvador apon- 
tou presença de dor crônica em $41,4 \%$ da população, sendo que esta predominava em mulheres $(48,3 \%)$, idosos, obesos, fumantes e ex-fumantes ${ }^{18}$.

A literatura mundial acusa maior porcentagem de dor em mulheres e idosos, justamente a população pesquisada no presente estudo. Avaliando os resultados, nota-se que nossa população apresentou valores superiores e quando os grupos foram comparados houve diferença estatística, mostrando maior ocorrência no grupo de nível socioeconômico mais baixo. Há trabalhos publicados associando condição socioeconômica, nível de escolaridade, situação conjugal e prática de atividade física com dor crônica, sendo que a situação de baixa renda, assim como o nível de escolaridade baixo, pode ser considerada como fator preditor para o desenvolvimento da dor crônica ${ }^{1920,21}$. A carga excessiva de trabalho das mulheres de baixa renda é uma realidade no Brasil. Muitas trabalham fora em atividades que exigem esforço físico, como empregadas domésticas, diaristas, vendedoras, caixas e quando chegam em casa ainda precisam realizar as tarefas domésticas, como limpeza, alimentação, cuidado com filhos e arrumação. Somado a isso muitas moram nas periferias e enfrentam horas no transporte público para efetuarem seus deslocamentos. Dentre as mulheres de baixa renda há muitas que cuidam de netos para que filhas/filhos ou noras/genros possam trabalhar. Todas essas situações, muito comuns na população que reside na periferia, podem acabar gerando sobrecarga física e emocional, que podem favorecer o aparecimento das dores crônicas, fato evidente em nossa pesquisa. Nas classes de maior renda essa condição é diferente e as mulheres mais velhas estão utilizando a maturidade para cuidar de si mesmas, não assumindo papéis que as afastem do autocuidado. Academia, viagens e tratamentos estéticos estão entre as práticas comuns nas mulheres idosas com poder aquisitivo. $\mathrm{O}$ grupo A de nossa pesquisa pode estar dentro deste contexto, já que são mulheres que participam semanalmente de encontros para estudarem variados assuntos dentro de uma universidade privada aberta à maturidade.

\section{INCONTINÊNCIA URINÁRIA (IU)}

As mulheres do grupo B apresentaram mais ca- sos de IU e constipação intestinal do que as do grupo A. Um estudo feito na região Nordeste do Brasil encontrou resultados semelhantes, comparando mulheres atendidas pela rede particular e rede pública ${ }^{10}$. No setor público, 26,9\% apresentaram IU e, no setor privado, 14,4\% ( $p=0,03$ ). Os grupos da presente pesquisa seguiram essa tendência, sendo que $41,2 \%$ das mulheres do grupo A e $65,2 \%$ do grupo B apresentaram IU. Mas a literatura é escassa de estudos que demonstrem evidências de maior risco de IU em relação ao nível socioeconômico. Outros dados podem estar relacionados a esse achado, como a maior taxa de partos via fórceps no grupo B ( $p$ $=0,016$ ) e número de partos normais e gestações que, apesar de não apresentarem diferença estatística, eram maiores no grupo B. Gestações podem ser fator de risco para IU pela sobrecarga que causa no assoalho pélvico. $\mathrm{O}$ mesmo ocorre com os partos, sendo que há maior risco de desenvolvimento de IU em mulheres que já pariram, tanto via vaginal como via cesárea quando comparadas às nulíparas. Vale ressaltar que parto vaginal, isoladamente, não deve ser encarado como causador de IU, mas sim se for associado a lesões do assoalho pélvico ${ }^{22,23}$.

\section{OBESIDADE}

Apesar da maioria das mulheres da pesquisa não apresentarem índice de massa corporal (IMC) elevado, houve associação de obesidade com IU dos tipos mista e de urgência. Esse achado não apresentou relação com nível socioeconômico, diferente de alguns estudos que relatam maior prevalência de obesos em populações mais carentes $^{24}$. A obesidade é conhecida como um dos principais fatores de risco para o aparecimento de disfunções do assoalho pélvico. Provavelmente isso ocorra como consequência do aumento de pressão intra-abdominal provocado pelo aumento de peso na região abdominal e, consequentemente, do aumento da pressão intravesical alterando o mecanismo do trato urinário ${ }^{25}$.

\section{FALTA DE LIBIDO}

Disfunções sexuais são comuns na população feminina idosa brasileira. Estudo feito em centro de convivência para idosos mostrou que para as idosas pes- 
quisadas a sexualidade estava relacionada com a saúde, estética e aspectos psicoafetivos e que as alterações ocorridas em decorrência do processo de envelhecimento, tanto no homem quanto na mulher, podem prejudicar o prazer sexual. Para que não haja prejuízo nas relações afetivas, é necessária uma adaptação às mudanças desta fase. Na velhice problemas comuns, como os decorrentes do desgaste do organismo, doenças e problemas familiares, podem causar dificuldades sexuais ${ }^{26}$. No presente estudo $46,9 \%$ das mulheres do grupo A e $75 \%$ do grupo $\mathrm{B}$ apresentaram falta de libido, sendo que os valores do grupo B foram significativamente maiores que o grupo A, sugerindo que componentes socioeconômicos possam influenciar negativamente a satisfação sexual.

A sexualidade humana está ligada à afetividade, ao prazer, intimidade, reprodução e também à autoestima e autoimagem. A forma de vivenciar a sexualidade pode ocorrer por pensamentos, relacionamentos e crenças, estando regulada por fatores biopsicossociais, culturais e econômicos. Muitas vezes a sexualidade é relacionada a fatores biológicos, deixando os outros aspectos com menor importância. As mulheres estudadas encontravam-se justamente na faixa etária onde a atividade sexual tende a diminuir, tanto pelo efeito da queda hormonal provocada pela menopausa quanto por fatores culturais. Crenças e valores muitas vezes tendem, erroneamente, a colocar o envelhecimento como uma fase da vida onde as mulheres não precisam ou não devem mais se relacionar sexualmente ou manter sua sexualidade viva.

Há um estigma em relação à sexualidade, que é considerada avessa à velhice e muitas vezes relacionada somente com juventude, sensualidade, fertilidade e procriação. Contudo a insatisfação sexual pode estar relacionada às dificuldades de desempenho sexual durante a velhice, que neste caso é de novo centrado apenas no ato sexual ${ }^{27}$. A falta de libido apresentada pelas idosas aqui estudadas poderia estar relacionada a um conformismo, alimentado por fatores culturais e educativos típicos da faixa etária que elas se encontram. A menopausa deveria ser encarada como um acontecimento natural da vida da mulher, mas frequentemente se apresenta de forma complexa em relação aos conhecimentos e sentidos, sendo acompanhada de conotações negativas, que a levam a ser identificada como doença. Menegon verificou em sua dissertação o uso de repertórios de conversas cotidianas e da literatura científica que naturalizam a menopausa como um problema. Essa naturalização poderia ser causada por alguns fatores: processo de medicalização, relações de gênero e poder e concepção negativa do envelhecimento na cultura ocidental. A autora reforça a necessidade de uma desconstrução de discursos, científicos ou não, que identifiquem a menopausa como período de sofrimento e que limitam a possibilidade de criação de novos sentidos para esse momento da vida das mulheres $^{28}$. Programas que abordem a sexualidade para o público mais velho são importantes para quebrar o paradigma que alimenta a ideia que a ausência de sexualidade seja inerente ao envelhecimento.

\section{CONHECIMENTO E REPERCUSSÃO DOS EXERCÍCIOS DO ASSOALHO PÉLVICO}

O programa de exercícios oferecido às mulheres desta pesquisa deixou claro que há falta de conhecimento em relação ao que é, onde se localiza e para que serve o assoalho pélvico, independentemente do nível socioeconômico, mas com pior nível de conhecimento nas classes menos favorecidas. Esta é uma realidade que reflete a falta de informação generalizada em relação ao corpo e autocuidado que experimentamos em nossa sociedade, onde os currículos educacionais não contemplam disciplinas voltadas a essa temática. A própria Educação Física, ministrada nas escolas, prioriza a performance específica de determinados gestos esportivos, muitas vezes excluindo aqueles que não possuem essas habilidades, o que pode interferir na falta de conhecimento e percepção corporal na vida adulta. Nesse sentido Daólio propôs uma Educação Física por ele denominada plural, onde os elementos da cultura corporal serão tratados como conhecimentos a serem sistematizados e reconstruídos pelos alunos ${ }^{29}$.

As mulheres que participaram deste estudo, pela própria faixa etária, não passaram por processos educacionais escolares com essa visão integral do corpo e muitas, possivelmente, nem tiveram a disciplina de Educação Física. Nas mulheres de nível socioeconômico mais baixo esse fator estava mais presente, devido à pouca escolaridade. Além das dificuldades com o acesso a uma Educa- 
ção Física mais pluralista, o desenvolvimento do conhecimento corporal se agrava quando falamos da região pélvica, que envolve sexualidade e, portanto, muito tabu.

Os resultados desta pesquisa sugerem que esse quadro pode ser modificado quando a informação é oferecida de forma simples, prática e clara. Após a intervenção com o método de exercícios em grupo, 32,4\% das mulheres do grupo A e 70,6\% das mulheres do grupo B referiram que aprenderam o que era o assoalho pélvico, e $67,6 \%$ das mulheres do grupo A e $29,4 \%$ do grupo B afirmaram que após as aulas o seu nível de conhecimento melhorou em relação a essa parte do corpo, um resultado estatisticamente significante, principalmente para o grupo de mulheres de nível socioeconômico mais baixo.

Além do aprendizado em relação ao assoalho pélvico houve melhora no autoconhecimento em relação à respiração, postura e consciência corporal, em ambos os grupos.

Exercícios em grupo são uma excelente estratégia para trabalhar a saúde de forma eficiente, com baixo custo e qualidade. Os grupos são executados de forma coletiva e interdisciplinar, estimulando os participantes a exercitar autonomia, autocuidado e desenvolvimento contínuo do nível de saúde e das condições de vida. O grupo não pode ser considerado simplesmente um agrupamento de pessoas, pois além das atividades propostas em si, há também apoio socioemocional e fortalecimento das relações pessoais, comunicação aberta, compromisso e responsabilidade ${ }^{30}$. Essas características puderam ser observadas em ambos os grupos desta pesquisa, reforçando a ideia de grupo como estratégia eficaz de intervenção. A aprovação do método e a sugestão da continuidade do programa por $71,6 \%$ das mulheres traz a possibilidade da replicação da metodologia em grupos distintos.

\section{CONCLUSÕES}

Essa pesquisa mostrou diferenças sociodemográficas e clínicas entre mulheres de diferentes níveis socioeconômicos da cidade de São Paulo que participaram de um programa de exercícios em grupo para assoalho pélvico, postura, respiração e consciência corporal. As mulheres de nível socioeconômico mais baixo relataram mais dor, incontinência urinária, constipação, maior número de partos via fórceps e mais falta de libido que as de nível socioeconômico mais alto. Já as mulheres do grupo de alto nível socioeconômico apresentaram mais casos de cirurgias ao longo da vida. Obesidade foi relacionada a uma maior probabilidade de desenvolvimento de incontinência urinária nos dois grupos, independentemente do nível socioeconômico.

Há falta de conhecimento sobre o assoalho pélvico nos dois grupos, mas em maior proporção no grupo de nível socioeconômico mais baixo.

O programa de exercícios em grupo promoveu melhora dos sintomas de perda urinária, consciência do corpo, da postura, da respiração e do assoalho pélvico em mulheres idosas de diferentes níveis socioeconômicos, sugerindo que estes possam ser utilizados como ferramenta de promoção de saúde por profissionais da área.

\section{AGRADECIMENTOS}

À Pontifícia Universidade Católica de São Paulo (PUC-SP) pelo auxílio para capacitação docente oferecido pelo Plano de Apoio à Pesquisa (Pipeq) à autora Juliana Schulze Burti, doutoranda pela instituição.

\section{REFERÊNCIAS}

1. Steele SR, Varma MG, Prichard D, Bharucha E, Vogler SA, Erdogan A et al. The evolution of evaluation and management of urinary or fecal incontinence and pelvic organ prolapse. Curr Probl Surg. 2015 Feb; 52 (2):17-75.

2. McClurg D, Pollock A, Campbell P, Hazelton C, Elders A, Hagen S, Hill DC. Conservative interventions for urinary incontinence in women: an Overview of Cochrane systematic reviews. Cochrane Database of Systematic Reviews 2016, 9.

3. Abrams P, Cardozo L, Khoury S, Wein A. Incontinence: 5 th international consultation on incontinence. Paris: International Continence Society; 2013. 
4. Faria CA, Menezes AMN, Rodrigues AO, Ferreira ALV, Bolsas CN. Incontinência urinária e noctúria: prevalência e impacto sobre qualidade de vida em idosas numa unidade básica de saúde. Rev Bras Geriatr Gerontol. 2014; 17(1):17-25.

5. Burti JS, Santos AM, Pereira RMR, Zambon JP, Marques AP. Prevalence and clinical characteristics of urinary incontinence in elderly individuals of a low income. Arch Gerontol Geriatr. 2012;54(2):42-6.

6. Langoni CS, Knorst MR, Lovatel GA, Leite VO, Resende TL. Incontinência urinária em idosas de Porto Alegre: sua prevalência e sua relação com a função muscular do assoalho pélvico. Fisioter Pesqui. 2014; 21(1):74-80.

7. Tamanini JT, Santos JL, Lebrão ML, Duarte YA, Laurenti R. Association between urinary incontinence in elderly patients and caregiver burden in the city of São Paulo/Brazil: health, wellbeing, and ageing study. Neurourol Urodyn. 2011; 30(7):1281-5.

8. Silva L; Lopes, MHBM. Incontinência urinária em mulheres: razões da não procura por tratamento. Rev. esc. enferm. USP. 2009 mar; 43(1):72-78.

9. 9 Guarisi T, Pinto-Neto AM, Osis MJ, Pedro AO, Costa-Paiva LHS, Faúndes A. Procura de Serviço Médico por mulheres com incontinência urinária. Rev Bras Ginecol Obstet. 2001; 23(7):439-443.

10. Prado DS, Lima TIA, Mota VPLP. Conhecimento sobre incontinência urinária em dois grupos de mulheres de diferentes níveis socioeconômicos. Rev Bras Cien Med Saúde. 2013; 2(2):12-17.

11. Varmus H. Disease-specific estimates of direct and indirect costs of illness and NIH support. 1997; Washington: Bethesda

12. Subak LL, Brown JS, Kraus SR, et al. The "Costs" of Urinary Incontinence for Women. Obstetrics and gynecology. 2006; 107(4):908-916.

13. National Institute for Healthcare and Excellence. The management of urinary incontinence in women. NICE clinical guideline. 2013 sep; (2):74-110.

14. Dumoulin C, Hay-Smith EJC, Mac Habée-Séguin G. Pelvic floor muscle training versus no treatment, or inactive control treatments, for urinary incontinence in women. Cochrane Database of Systematic Reviews. 2014;14(5).

15. Alves F, Riccetto C, Adami DB, Marques J, Pereira LC, Palma $\mathrm{P}$, et al. A pelvic floor muscle training program in postmenopausal women. A randomized controlled trial. Maturitas 2015; 81(2):300-5.

16. Definição e Metodologia de Cálculo dos Indicadores e Índices de Desenvolvimento Humano e Condições de Vida. Rio de Janeiro: IPEA, 1999.

17. IBGE/Censo 2000 e Fundação SEADE. Desigualdade em São Paulo: o IDH. Elaboração SDTS/PMSP, 2002.

18. Sá K, Baptista AF, Matos MA, Lessa I. Prevalência de dor crônica e fatores associados na população de Salvador, Bahia. Rev Saúde Publica, 2009;43(4):622-630.

19. Turner JA, Franklin G, Fulton-Kehoe D, Egan K, Wickizer TM, Lymp JF, et al. Prediction of chronic disability in work-related musculoskeletal disorders: a prospective, population-based study. BMC Musculoskelet Disord. 2004;5:14.

20. Hirano K, Imagama S, Hasegawa Y, Ito Z, Muramoto A, Ishiguro N. Impact of low back pain, knee pain, and timed up-and-go test on quality of life in community-living people. J Orthop Sci. 2014; 19(1):164-71.

21. Pieretti S, Di Giannuario A, Di Gionavvavdrea R, Marzoli F, Piccaro G, Minosi P, et al. Gender differences in pain and its relief. Ann Ist Super Sanita. $2016 \mathrm{Apr}-$ Jun; 52(2):184-9.

22. Haylen BT, Maher CF, Barber MD, Camargo S, Dandolu V, Digesu A, et al. An international urogynecological association (IUGA)/International continence society joint report on the terminology for female pelvic organ prolapse. International Urogynecology Journal. 2016; 27(2):165-94.

23. 23. Almousa S, Van Loon AB. The prevalence of urinary incontinence in nulliparous adolescent and middle-aged women and the associated risk factors: A systematic review. Maturitas. 2018 January; 107, 78-83.

24. Gigante D P et al. Obesidade da população adulta de Pelotas, Rio Grande do Sul, Brasil e associação com 
nível sócio-econômico. Cad. Saúde Pública. 2006 set; 22(9):1873-1879.

25. Bolina AF, Dias FA, Santos NMF, Tavares DMS. Incontinência Urinária autorreferida em idosos e seus fatores associados. Revista da Rede de Enfermagem do Nordeste. 2013; 14(2):354-63.

26. Costa RB, Sousa KKO, Vasconcelos RS, Câmara TMS, Vasconcelos TB, Bastos VPD. Sexualidade em idosas participantes de um grupo de convivência. Saúde e Pesquisa. 2015; 8(2):239-45.

27. Santos DLR, Faustino AM. Saúde sexual e sexualidade de mulheres idosas: revisão de literatura. Rev Gestão \& Saúde 2017;1(3):674-91.

28. Menegon VSM. Menopausa: imaginário social e conversas do cotidiano. [dissertação de mestrado]. São Paulo: Pontifícia Universidade Católica de São Paulo - PUC-SP; 1998.

29. Daolio, J. Educação Física Escolar: em busca da Pluralidade. Rev. paul. Educ. Fis. 1996;2:40-42.

30. Recco, RAC e Lopes, SMB. Sobre Fisioterapia e seus recursos terapêuticos: o grupo como estratégia complementar à reabilitação. Trab. Educ. Saúde 2016 maio/ago; 14(2):593-610.

Recebido em: 30/07/2018

Aceito em: 09/10/2018 